\title{
Neoadjuvant Therapy for Rectal Cancer Affects Lymph Node Yield and Status Without Clear Implications on Outcome: The Case for Eliminating a Metric and Using Preoperative Staging to Guide Therapy
}

\author{
Sherif R. Z. Abdel-Misih, MDª Lai Wei, $\mathrm{PhD}^{\mathrm{b}}$; Al B. Benson III, MD; ; Steven Cohen, MD; Lily Lai, MDe; \\ John Skibber, MDf; Neal Wilkinson, MD, MPH'; Martin Weiser, MD ${ }^{\text {h }}$; Deborah Schrag, MD, MPHi; and \\ Tanios Bekaii-Saab, MDj
}

\begin{abstract}
Background: Nodal status has long been considered pivotal to oncologic care, staging, and management. This has resulted in the establishment of rudimentary metrics regarding adequate lymph node yield in colon and rectal cancers for accurate cancer staging. In the era of neoadjuvant treatment, the implications of lymph node yield and status on patient outcomes remains unclear. Patient and Methods: This study included 1,680 patients with locally advanced rectal cancer from the NCCN prospective oncology database stratified into 3 groups based on preoperative therapy received: no neoadjuvant therapy, neoadjuvant chemoradiation, and neoadjuvant chemotherapy. Clinicopathologic characteristics and survival were compared between the groups, with univariate and multivariate analyses undertaken. Results: The clinicopathologic characteristics demonstrated statistically significant differences and heterogeneity among the 3 groups. The neoadjuvant chemoradiation group demonstrated the statistically lowest median lymph node yield $(n=15)$ compared with 17 and 18 for no-neoadjuvant and neoadjuvant chemotherapy, respectively $(P<.0001)$. Neoadjuvant treatment did impact survival, with chemoradiation demonstrating increased median overall survival of 42.7 compared with 37.3 and 26.6 months for neoadjuvant chemotherapy and noneoadjuvant therapy, respectively $(P<.0001)$. Patients with a yield of fewer than 12 lymph nodes had improved median overall survival of 43.3 months compared with 36.6 months in patients with 12 or more lymph nodes $(P=.009)$. Multivariate analysis demonstrated that neither node yield nor status were predictors for overall survival. Discussion: This analysis reiterates that nodal yield in rectal cancer is multifactorial, with neoadjuvant therapy being a significant factor. Node yield and status were not significant predictors of overall survival. A nodal metric may not be clinically relevant in the era of neoadjuvant therapy, and guidelines for perioperative therapy may need reconsideration.
\end{abstract}

J Natl Compr Canc Netw 2016;14(12):1528-1534

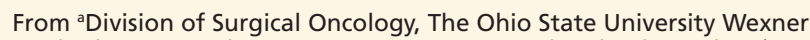
Medical Center/Arthur G. James Cancer Hospital, Columbus, Ohio; ${ }^{b}$ Center for Biostatistics, The Ohio State University Wexner Medical Center, Columbus, Ohio; 'Robert H. Lurie Comprehensive Cancer Center of Northwestern University, Chicago, Illinois; 'Department of Hematology/ Oncology, Fox Chase Cancer Center, Philadelphia, Pennsylvania; ${ }^{e}$ City of Hope Comprehensive Cancer Center, Duarte, California; ${ }^{\text {fUniversity of }}$ Texas MD Anderson Cancer Center, Houston, Texas; ${ }^{9}$ Kalispell Regional Healthcare Specialists, Kalispell Regional Healthcare, Kalispell, Montana;

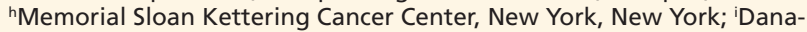
Farber Cancer Institute, Boston, Massachusetts; and iDepaartment of Medicine, Division of Hematology/Oncology, Mayo Clinic Cancer Center, Phoenix, Arizona.

Submitted July 1, 2016; accepted for publication August 17, 2016.

The authors have disclosed that they have no financial interests, arrangements, affiliations, or commercial interests with the manufacturers of any products discussed in this article or their competitors.
Author Contributions: Bekaii-Saab and Abdel-Misih formulated the scientific question (concept) and the associated data points of interest to be obtained from the NCCN cancer database. Schrag reviewed the initial concept with approval for use of the NCCN cancer database for this scientific inquiry. LW was involved in the statistical concepts and analysis of the acquired data. The qualitative data analysis, interpretation, and drafting of the manuscript were undertaken by Abdel-Misih and BekaiiSaab. All authors (Weiser, Benson, Cohen, Lai, Skibber, Wilkinson, Weiser, Schrag, Bekaii-Saab, and Abdel-Misih) contributed to the review and editing of the manuscript. All authors had final approval of the version to be published and are in agreement to be accountable for all aspects of the work in ensuring questions related to the accuracy and integrity of the work are appropriately investigated and resolved.

Correspondence: Sherif R. Z. Abdel-Misih, MD, 320 West 10th Avenue, M256 Starling-Loving Hall, Columbus, OH 43210.

E-mail: sherif.abdel-misih@osumc.edu 


\section{Background}

Nodal status has long been considered a key pathologic factor in cancer management and care. Previous studies have demonstrated an association between lymph node yield and status with regard to patient outcomes in colorectal cancer (CRC)..$^{1-4}$ As such, it was first recommended at the World Congress of Gastroenterology in 1990 that at least 12 lymph nodes be examined after radical resection to optimally stage patients, and the impact of preoperative therapy on nodal yield was acknowledged. ${ }^{5}$ Subsequently, several major organizations, including the American Joint Committee on Cancer (AJCC), Union for International Cancer Control, and College of American Pathologists (CAP) have recommended at least 12 lymph nodes be examined for accurate staging in CRC becoming a well-accepted metric.

Since that time, several large population-based studies have demonstrating inadequate lymph node harvest and evaluation being undertaken in CRC. ${ }^{6,7}$ This has been more prevalent with rectal cancer, and a number of studies have determined limitations to this, including variability related to surgical techniques, pathologic assessment, patient factors, institutions, and the effect of neoadjuvant therapy. ${ }^{8-12}$

Regarding surgical technique, it is well accepted that a total mesorectal excision (TME) should be undertaken to help achieve optimal oncologic outcomes. Proficient TME allows for harvest of the traditional primary nodal drainage basin and optimizes the circumferential resection margin. If an incomplete TME is undertaken, this can account for "understaging" ( $\mathrm{Nx}$ ) with inadequate surgical lymph node yield, and could potentially negatively impact the outcome with disease recurrence.

Pathologic techniques have been implicated as a factor in lymph node yield due to inadequate specimen analysis and processing. Patient-related factors, such as age and obesity, have been associated with decreased lymph node yield. ${ }^{13-16}$ Institutional-related factors demonstrate variation and high noncompliance with the 12 -or-more lymph node metric. ${ }^{17,18}$

In the past decade, the standard of care for rectal cancer has been based on the German Rectal Cancer Study Group trial. ${ }^{19}$ This phase III trial compared neoadjuvant chemoradiation with the thenstandard postoperative chemoradiation therapy and demonstrated a statistically significant improvement in local recurrence with reduced treatment toxicity, but without a difference in overall survival (OS). Because neoadjuvant chemoradiation was established as the standard of care for clinical stage II/III rectal cancer, studies have demonstrated its' association with decreased lymph node yield..$^{20-22}$

Recently, long-term results from the EORTC 22921 study examined the utility of fluorouracilbased adjuvant chemotherapy after preoperative therapy for rectal cancer. ${ }^{23}$ This study concluded that adjuvant chemotherapy demonstrated no statistically significant improvement in disease-free survival or OS in patients after neoadjuvant treatment. This naturally prompts the question as to the utility of the current standard of care, which is 6 months of perioperative systemic therapy.

Given the unclear utility of adjuvant therapy after preoperative therapy has been administered, the clinical importance of pathologic nodal yield and status must be questioned given their impact on the treatment decisions made. Hence, an established lymph node metric and nodal status for rectal cancer may or may not be clinically relevant in the era of neoadjuvant chemoradiation administered for locally advanced rectal cancer.

\section{Patients and Methods}

NCCN is a not-for-profit alliance comprising 27 of the nation's leading cancer centers whose goal is to improve the quality of multidisciplinary cancer care for patients. NCCN has compiled a prospective NCCN Oncology Outcomes Database for CRCs abstracted from 8 participating NCCN institutions: City of Hope Comprehensive Cancer Center (CCC), Memorial Sloan Kettering Cancer Center, The University of Texas MD Anderson Cancer Center, Dana-Farber Cancer Institute, Fox Chase Cancer Center, Roswell Park Cancer Center, Robert H. Lurie CCC at Northwestern University, and The Ohio State University CCC - James Cancer Hospital and Solove Research Institute. The data collection process was approved by the respective institutions' Institutional Review Boards.

A cohort was compiled of 1,680 patients with clinical stage II/III primary rectal cancer who underwent surgical intervention excluding pelvic exenteration, subtotal colectomy, total proctocolectomy, transanal excision, Hartmann's procedure, and recto- 
sigmoid resections. The patient cohort was divided into 3 groups based on the type of neoadjuvant therapy received before surgery: no-neoadjuvant therapy $(n=111)$, neoadjuvant chemoradiation $(n=1,364)$, and neoadjuvant chemotherapy $(n=205)$. This analysis is inclusive of data from September 2005 to September 2013.

The clinicopathologic characteristics were analyzed and compared using chi-square test for categorical variables and the Kruskal-Wallis test for continuous variables among the 3 groups. Lymph node analysis for group comparisons was undertaken using the Kruskal-Wallis test. Clinicopathologic characteristics effect on lymph node were analyzed using the Kruskal-Wallis or Wilcoxon test as appropriate.

OS was defined from date of diagnosis to date of death. Patients alive at the date of last observation were censored for survival analysis. Survival curves were estimated using the Kaplan-Meier method. Survival curves were compared using the log-rank tests for the univariate analysis. Multivariable Cox regression models were fit to the $\mathrm{OS}$ using all the variables with a $P$ value of less than 0.2 in the univariate analysis. Variables with a $P$ value greater than 0.05 were removed sequentially from the Cox regression model. All statistical analyses were conducted using SAS 9.2 (SAS Institute Inc., Cary, NC).

\section{Results}

The 3 groups' clinicopathologic characteristics were compared as illustrated in Table 1, showing heterogeneity between groups, with statistical significance demonstrated for median age, age at diagnosis, Charlson comorbidity index, pathologic TNM stage, type of surgery undertaken, median OS, lymph node yield, and node positivity.

The treatment groups demonstrated statistical significance with regard to median age and age at diagnosis. The no-neoadjuvant group had an increased median age of 64 years, compared with 57 and 56 years for neoadjuvant chemoradiation and the neoadjuvant chemotherapy groups, respectively $(P<.0001)$. Likewise, the age of diagnosis in the no-neoadjuvant group demonstrated a higher proportion of patients aged 65 years or older at 45\% compared with $26 \%$ and $28 \%$ in the neoadjuvant chemoradiation and neoadjuvant chemotherapy groups, respectively $(P=.0001)$. In examining the comorbidities of the group, the noneoadjuvant therapy group had a higher proportion of patients with a Charlson comorbidity index greater than 2 , at $16 \%$ compared with $8 \%$ and $10 \%$ in the neoadjuvant chemoradiation and neoadjuvant chemotherapy groups, respectively $(P=.02)$.

Statistically significant differences in pathologic staging were seen among the 3 groups, with the neoadjuvant chemoradiation group showing a greater proportion of patients with pathologic stage 0 disease: 20\% compared with $5 \%$ and $9 \%$ in the no-neoadjuvant and neoadjuvant chemotherapy groups, respectively. Pathologic stage 3 disease was seen in 30\% of patients in the neoadjuvant chemoradiation group compared with $60 \%$ and $15 \%$ in the neoadjuvant chemotherapy and no-neoadjuvant therapy groups, respectively.

The neoadjuvant chemoradiation group demonstrated the lowest median lymph node yield at 15 compared with 17 and 18 in the no-neoadjuvant and neoadjuvant chemotherapy groups, respectively $(P<.0001)$. In analyzing the number of positive nodes between groups, a statistically significant difference was seen, with the neoadjuvant chemotherapy group demonstrating increased positive nodes at a mean of $2.2 \pm$ 3.6 compared with $0.9 \pm 2.1$ and $0.8 \pm 4.4$ for the neoadjuvant chemoradiation and no-neoadjuvant groups $(P<.0001)$. In comparing median OS, improved survivals of 42.7 and 37.3 months were seen in the neoadjuvant chemotherapy and neoadjuvant chemoradiation groups, respectively, compared with 26.6 months in the no-neoadjuvant therapy group $(P<.0001)$.

As illustrated in Table 2, univariate analysis demonstrated that the factors associated with an effect on lymph node yield included tumor grade, clinical $\mathrm{T}$ stage, surgery type, and neoadjuvant chemoradiation. Multivariate analysis revealed that surgery type, tumor grade, clinical $\mathrm{T}$ stage, and neoadjuvant treatment were predictors of OS, with neither node number nor status being statistically significant. When examining nodal status irrespective of nodal yield, patients with positive lymph node status, although not statistically significant, had an increased OS of 38.4 months compared with 36.9 months for node-negative status $(P=.09)$.

Subgroup analyses were undertaken examining the impact of both nodal yield and status on OS, as illustrated in Table 3 and Figure 1. Patients with a lymph node yield of less than 12 had an improved median OS of 43.3 months compared with 36.6 months in patients with 12 or more lymph nodes $(P=.009)$. Further analysis of patients with a 
Nodal Impact After Neoadjuvant Therapy

\begin{tabular}{|c|c|c|c|c|c|}
\hline Variable & Category & $\begin{array}{c}\text { No Neoadjuvant } \\
(\mathrm{N}=111)\end{array}$ & $\begin{array}{c}\text { Neoadjuvant } \\
\text { Chemoradiation } \\
(\mathrm{N}=1,364)\end{array}$ & $\begin{array}{c}\text { Neoadjuvant } \\
\text { Chemotherapy } \\
\text { Only } \\
(\mathrm{N}=205) \\
\end{array}$ & $P$ Value \\
\hline Median a & age, y & 64 (38-93) & 57 (19-89) & $56(25-89)$ & $<.0001$ \\
\hline \multicolumn{6}{|c|}{ Age at diagnosis } \\
\hline & $<65 y$ & $61(55 \%)$ & $1,004(74 \%)$ & $148(72 \%)$ & \multirow[t]{2}{*}{.0001} \\
\hline & $\geq 65 y$ & $50(45 \%)$ & $360(26 \%)$ & $57(28 \%)$ & \\
\hline \multicolumn{6}{|l|}{ Sex } \\
\hline & Male & $58(52 \%)$ & $813(60 \%)$ & $111(54 \%)$ & \multirow[t]{2}{*}{.13} \\
\hline & Female & $53(48 \%)$ & $551(40 \%)$ & $94(46 \%)$ & \\
\hline \multicolumn{6}{|c|}{ Charlson comorbidity } \\
\hline & 0 or 1 & $93(84 \%)$ & $1249(92 \%)$ & $184(90 \%)$ & \multirow[t]{2}{*}{.02} \\
\hline & $\geq 2$ & $18(16 \%)$ & $115(8 \%)$ & $21(10 \%)$ & \\
\hline \multicolumn{6}{|c|}{ Pathologic TNM stage } \\
\hline & 0 & $5(5 \%)$ & $272(20 \%)$ & $19(9 \%)$ & \multirow[t]{4}{*}{$<.0001$} \\
\hline & 1 & $57(51 \%)$ & $359(26 \%)$ & $13(6 \%)$ & \\
\hline & II & $32(29 \%)$ & $324(24 \%)$ & $51(25 \%)$ & \\
\hline & III & $7(15 \%)$ & $409(30 \%)$ & $122(60 \%)$ & \\
\hline \multicolumn{6}{|c|}{ Type of surgery } \\
\hline & LAR & $90(81 \%)$ & $952(70 \%)$ & $175(85 \%)$ & \multirow[t]{3}{*}{$<.0001$} \\
\hline & APR & $15(14 \%)$ & $332(24 \%)$ & $26(13 \%)$ & \\
\hline & Proctectomy & $6(5 \%)$ & $80(6 \%)$ & $4(2 \%)$ & \\
\hline \multicolumn{6}{|c|}{ Adjuvant chemotherapy } \\
\hline & Yes & $0(0 \%)$ & $1,364(100 \%)$ & $205(100 \%)$ & \multirow[t]{2}{*}{.0001} \\
\hline & No & $111(100 \%)$ & $0(0 \%)$ & $0(\%)$ & \\
\hline \multicolumn{6}{|c|}{ NCCN institution ${ }^{\mathrm{a}}$} \\
\hline & $\mathrm{A}$ & $12(11 \%)$ & $66(5 \%)$ & $8(4 \%)$ & \multirow{8}{*}{$\begin{array}{c}\text { Not } \\
\text { compared }\end{array}$} \\
\hline & B & $15(14 \%)$ & $111(8 \%)$ & $24(12 \%)$ & \\
\hline & $\mathrm{C}$ & $4(4 \%)$ & $70(5 \%)$ & $3(1 \%)$ & \\
\hline & D & $24(22 \%)$ & $351(26 \%)$ & $42(20 \%)$ & \\
\hline & $\mathrm{E}$ & $4(4 \%)$ & $74(5 \%)$ & $11(5 \%)$ & \\
\hline & $\mathrm{F}$ & $30(27 \%)$ & $140(10 \%)$ & $26(13 \%)$ & \\
\hline & G & $11(10 \%)$ & $452(33 \%)$ & $85(41 \%)$ & \\
\hline & $\mathrm{H}$ & $11(10 \%)$ & $100(7 \%)$ & $6(3 \%)$ & \\
\hline Median C & $\mathrm{SS}(95 \% \mathrm{Cl}), \mathrm{mo}$ & $26.6(23.1,33.3)$ & $37.3(36.6,39.2)$ & $42.7(36.1,47.0)$ & $<.0001$ \\
\hline \multicolumn{6}{|c|}{ Lymph nodes removed } \\
\hline & Mean $\pm S D$ & $19.9 \pm 10.0$ & $16.3 \pm 7.0$ & $19.4 \pm 8.3$ & \\
\hline & $\begin{array}{l}\text { Median } \\
\text { (range) }\end{array}$ & $17(4-64)$ & $15(1-69)$ & $18(2-61)$ & $<.0001$ \\
\hline \multicolumn{6}{|c|}{ Positive lymph nodes } \\
\hline & Mean $\pm S D$ & $0.8 \pm 4.4$ & $0.9 \pm 2.1$ & $2.2 \pm 3.6$ & $<.0001$ \\
\hline & $\begin{array}{l}\text { Median } \\
\text { (range) }\end{array}$ & $0(0-44)$ & $0(0-16)$ & $1(0-28)$ & \\
\hline
\end{tabular}

Abbreviations: APR, abdominoperitoneal resection; LAR, low anterior resection; OS, overall survival.

${ }^{a}$ NCCN Member Institutions include Fox Chase Cancer Center, City of Hope Comprehensive Cancer Center, The University of Texas MD Anderson Cancer Center, Roswell Park Cancer Institute, Dana-Farber Cancer Institute, The Ohio State University Comprehensive Cancer Center-James Cancer Hospital and Solove Research Institute, Memorial Sloan Kettering Cancer Center, and Robert H. Lurie Comprehensive Cancer Center of Northwestern University.

lymph node yield of fewer than 12 revealed that those with positive nodal status had an increased median OS of 46.5 months compared with 41.5 months for negative nodal status, although not statistically significant $(P=.46)$. Similarly, patients with a lymph node yield of 12 or more and positive nodal status had an increased median OS of 37.4 months compared with 36.3 months in those with negative nodal status, with a trend that approaches but is not statistically significant $(P=.07)$. In examining patients receiving neoadjuvant chemoradiation, those with a lymph node yield of less than 12 had an improved OS of 43.4 months compared with 36.8 months in those with 12 or more lymph nodes $(P=.02)$.

\section{Discussion}

Lymph node status remains an essential factor for cancer staging and guiding treatment decisions. In 


\begin{tabular}{|c|c|c|c|c|c|c|}
\hline Variable & Category & $\mathbf{N}$ & $\begin{array}{l}\text { Median } \\
\text { OS (mo) }\end{array}$ & $95 \% \mathrm{Cl}$ & $\begin{array}{c}\text { Univariate } \\
\text { Analysis } \\
P \text { Value }\end{array}$ & $\begin{array}{c}\text { Multivariate } \\
\text { Analysis } \\
P \text { Value } \\
\end{array}$ \\
\hline \multicolumn{7}{|c|}{ Age at diagnosis } \\
\hline & $<50 y$ & 483 & 37.3 & $36.2-40.3$ & .70 & Not included \\
\hline & $50-64$ y & 730 & 37.3 & $35.9-40.1$ & & \\
\hline & $65-74 y$ & 318 & 36.8 & $34.6-40.0$ & & \\
\hline & $\geq 75 y$ & 149 & 37.9 & $33.1-44.8$ & & \\
\hline \multicolumn{7}{|l|}{ Sex } \\
\hline & Male & 982 & 38.4 & $36.9-40.9$ & .17 & .19 \\
\hline & Female & 649 & 36.4 & $35.2-37.5$ & & \\
\hline \multicolumn{7}{|c|}{ Charlson comorbidity } \\
\hline & 0 & 1,251 & 37.3 & $36.4-38.9$ & .30 & Not included \\
\hline & 1 & 275 & 40.9 & $35.1-45.5$ & & \\
\hline & $\geq 2$ & 154 & 36.4 & $33.1-40.3$ & & \\
\hline \multicolumn{7}{|c|}{ Pathologic TNM stage } \\
\hline & 0 & 296 & 37.7 & $35.7-41.2$ & .55 & Not included \\
\hline & I & 429 & 37.0 & $35.4-40.4$ & & \\
\hline & II & 407 & 36.8 & $34.6-39.5$ & & \\
\hline & III & 548 & 38.0 & $36.4-40.8$ & & \\
\hline \multicolumn{7}{|c|}{ Type of surgery } \\
\hline & LAR & 1,217 & 38.3 & $36.8-40.3$ & $<.0001$ & $<.001$ \\
\hline & APR & 373 & 36.7 & $35.1-40.4$ & & \\
\hline & Proctectomy & 90 & 34.9 & $27.1-36.2$ & & \\
\hline \multicolumn{7}{|c|}{ Tumor grade at diagnosis } \\
\hline & 1 & 81 & 44.6 & $36.9-52.3$ & .02 & .02 \\
\hline & II & 1,222 & 37.0 & $36.0-38.5$ & & \\
\hline & III & 109 & 42.0 & $37.2-49.4$ & & \\
\hline & Unknown & 268 & 36.0 & $32.4-38.6$ & & \\
\hline \multicolumn{7}{|c|}{ Clinical T stage $\mathrm{e}^{\mathrm{b}}$} \\
\hline & $\mathrm{Tx}$ & 206 & 36.8 & $32.6-40.6$ & .03 & .03 \\
\hline & $\mathrm{T} 0 / \mathrm{T} 1 / \mathrm{T} 2$ & 143 & 40.3 & $35.6-45.5$ & & \\
\hline & $\mathrm{T3}$ & 1,234 & 37.5 & $36.6-39.2$ & & \\
\hline & $\mathrm{T4}$ & 97 & 31.5 & $26.2-36.4$ & & \\
\hline \multicolumn{7}{|c|}{ Node status (lymph nodes positive) } \\
\hline & Negative & 1,144 & 36.9 & $35.7-38.5$ & .09 & .31 \\
\hline & Positive & 536 & 38.4 & $36.7-42.1$ & & \\
\hline \multicolumn{7}{|c|}{ Time from the last dose to surgery $(\mathrm{N}=500)$} \\
\hline & $\leq 8 \mathrm{wk}$ & 314 & 22.3 & $20.9-23.6$ & .25 & Not included \\
\hline & $>8 \mathrm{wk}$ & 186 & 17.9 & $15.9-22.2$ & & \\
\hline \multicolumn{7}{|c|}{ Adjuvant chemotherapy } \\
\hline & Yes & 1,569 & 37.7 & $36.8-39.5$ & $<.0001$ & Redundant $^{a}$ \\
\hline & No & 111 & 26.6 & $23.1-33.3$ & & \\
\hline \multicolumn{7}{|c|}{ Neoadjuvant treatment } \\
\hline & No neoadjuvant chemotherapy & 111 & 26.6 & $23.1-33.3$ & $<.0001$ & $<.001$ \\
\hline & Neoadjuvant chemoradiation & 1,364 & 37.3 & $36.6-39.2$ & & \\
\hline
\end{tabular}

Abbreviations: APR, abdominoperitoneal resection; LAR, low anterior resection; OS, overall survival.

aAdjuvant chemotherapy is redundant because all the patients who received chemotherapy neoadjuvantly received chemotherapy in the adjuvant setting

examining the factors associated with lymph node yield in rectal cancer, modifiable factors include surgical and pathology measures. Surgical vigilance with optimal oncologic surgical technique with TME is desired. However, despite the highly qualified surgeons from participating NCCN institutions, there remain some patients in which a lymph node yield of 12 or more was not achieved irrespective of neoadjuvant treatment, as shown in Table 1, with nodal yields ranging from 1 to 69 in this patient series.

CAP understands the importance of lymph node yield, as reflected in their suggested rectal specimen assessment protocols. This requires meticulous tissue assessment but is not necessarily standardized, with 


\begin{tabular}{|c|c|c|c|c|}
\hline Group Category & $\mathbf{N}$ & $\begin{array}{l}\text { Median OS } \\
\quad(\mathrm{mo})\end{array}$ & $95 \% \mathrm{Cl}$ & $P$ Value \\
\hline \multicolumn{5}{|l|}{ All groups } \\
\hline$<12$ nodes & 323 & 43.3 & $37.8-48.7$ & .009 \\
\hline$\geq 12$ nodes & 1,357 & 36.6 & $35.7-37.8$ & \\
\hline \multicolumn{5}{|l|}{ Lymph nodes $<12$} \\
\hline Negative & 249 & 41.5 & $36.9-48.9$ & .46 \\
\hline Positive & 74 & 46.5 & $37.5-51.5$ & \\
\hline \multicolumn{5}{|l|}{ Lymph nodes $\geq 12$} \\
\hline Negative & 895 & 36.3 & $35.1-37.3$ & .07 \\
\hline Positive & 462 & 37.4 & $36.0-40.8$ & \\
\hline \multicolumn{5}{|c|}{ Chemoradiation group } \\
\hline$<12$ nodes & 288 & 43.4 & $37.5-48.9$ & .02 \\
\hline$\geq 12$ nodes & 1,076 & 36.8 & $35.9-38.3$ & \\
\hline
\end{tabular}

Abbreviation: OS, overall survival.

technique variance in grossing, fixation, processing, embedding, staining, and, ultimately, pathologist review all potentially affecting lymph node yield. They acknowledge the challenges of nodal assessment, particularly in the elderly, obese, and patients treated neoadjuvantly.

Neoadjuvant therapy is a well-accepted nonmodifiable factor (with the exception of deferring it) affecting lymph node yield; this was again demonstrated in this analysis with statistical significance seen between groups, with the neoadjuvant chemoradiation group having the lowest median lymph node yield $(n=15)$. In examining the pathologic staging of the groups, the neoadjuvant chemoradiation group not only had a greater stage 0 (pathologic complete response) rate at $20 \%$ versus $9 \%$ in the neoadjuvant chemotherapy group, but also comprised 30\% with pathologic stage III disease versus $60 \%$ in the neoadjuvant chemotherapy group. This difference could be reflective of response to the neoadjuvant treatment given but may also relate to earlier clinical staging.

Again, EORTC 22921 concluded that fluorouracil-based adjuvant chemotherapy showed no statistically significant improvement in disease-free survival or OS in patients after neoadjuvant treatment. Among patients with clinical stage II/III rectal cancer who can tolerate multimodality therapy and receive neoadjuvant chemoradiation, the benefit of adjuvant therapy is unclear, and hence the clinical importance of nodal status, and particularly yield, remains in question.

This study has several limitations. This is a retrospective analysis undertaken using the NCCN Oncology Outcomes Database. The NCCN database

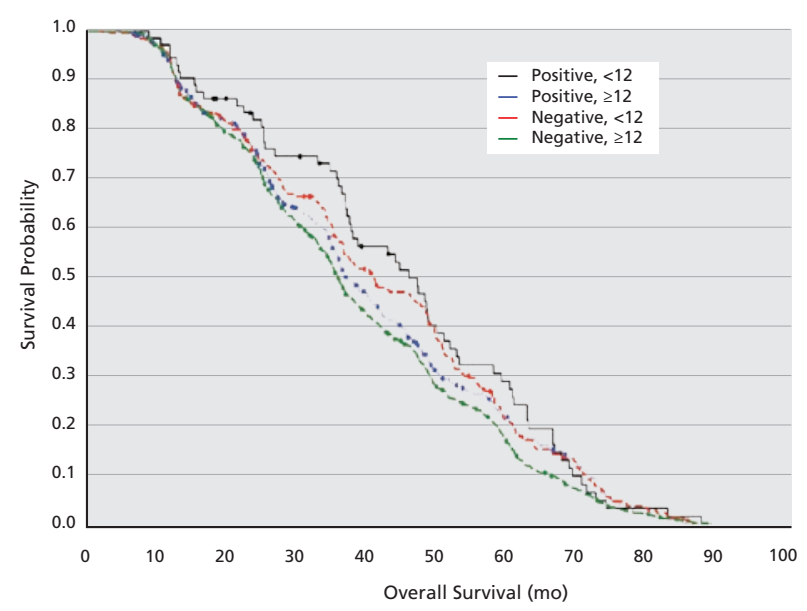

Figure 1. Overall survival stratified by nodal yield $(<12$ and $\geq 12)$ and status (negative/positive).

comprises a specific patient population derived from 8 participating NCCN Member Institutions, which constitute some of the leading tertiary care cancer institutions in the country. This certainly introduces several biases warranting mention. Given the specialized nature of NCCN treatment centers, this lends to potential referral bias such that the NCCN patient sample may not be truly representative of the overall patient population. Additionally, the specialized nature of NCCN Member Institutions introduces selection bias in the clinician expertise, patients treated, and the level and type of cancer care available and administered to this specific patient sample. This is exemplified in the group stratification based on therapy that is likely determined by patient characteristics and clinician recommendations despite equivalent preoperative clinical staging (clinical stage II/III).

As is often encountered with many large population database studies, the NCCN database has inherent limitations regarding the data available for analysis. There exists missing or even desired but unavailable data for which data imputation and analysis is not reasonable to pursue for scientifically sound statistics and conclusions, thus leaving some questions unanswered. Finally, as in all retrospective analyses, the factors examined and analyzed cannot be considered causative, but rather associated with the obtained results and drawn following conclusions.

Acknowledging the limitations of this large, but retrospective analysis, this analysis demonstrated no association between increased lymph node harvest or nodal status on patient survival irrespective of the patient therapies undertaken. The current NCCN 
Guidelines discussion based on previous studies acknowledges the impact of neoadjuvant therapy on decreased lymph node yield. ${ }^{24}$ This study interestingly demonstrated that patients with fewer than 12 lymph nodes harvested had improved OS irrespective of nodal status. Hence, a nodal metric in rectal cancer may not be clinically relevant in the setting of the current standard of care, with neoadjuvant treatment playing a major role in the multimodality treatment of rectal cancer.

Nodal yield and status after neoadjuvant therapy may more importantly be a surrogate marker of disease response and not ideal to base adjuvant therapy decisions on, particularly given the EORTC conclusions showing no demonstrable benefit to 5-FU-based adjuvant therapy. Preoperative clinical staging may in fact be more representative of cancer biology and therefore a more ideal characteristic on which to base adjuvant therapy decision-making to potentially impact and improve patient outcomes. That being said, in patients who were not significantly downstaged by neoadjuvant treatment and had positive lymph node status, one could anticipate potentially adverse outcomes based on their pathologic staging and lack of response to neoadjuvant therapy. Given the impact of neoadjuvant therapy on rectal cancer outcomes, ongoing (eg, NCCTG N1048 [PROSPECT]) and future prospective randomized trials are prudent and needed to further determine the relevance of lymph node yield and status to patient outcomes, and to optimize therapeutic approaches in order to advance the multimodality care of rectal cancer.

\section{References}

1. Le Voyer TE, Sigurdson ER, Hanlon AL, et al. Colon cancer survival is associated with increasing number of lymph nodes analyzed: a secondary survey of intergroup trial INT-0089. J Clin Oncol 2003;21;2912-2919.

2. Swanson RS, Compton CC, Stewart AK, Bland KI. The prognosis of $\mathrm{T} 3 \mathrm{~N} 0$ colon cancer is dependent on the number of lymph nodes examined. Ann Surg Oncol 2003;10:65-71.

3. Tepper JE, O'Connell MJ, Niedzwiecki D, et al. Impact of number of nodes retrieved on outcome in patients with rectal cancer. J Clin Oncol 2001;19:157-163.

4. Cserni G, Vinh-Hung V, Burzykowski T. Is there a minimum number of lymph nodes that should be histologically assessed for a reliable nodal staging of T3NOMO colorectal carcinomas? J Surg Oncol 2002;81:63-69.

5. Fielding LP, Arsenault PA, Chapuis PH, et al. Clinicopathologic staging for colorectal cancer: an International Documentation System (IDS) and an International Comprehensive Anatomical Terminology (ICAT). J Gastroenterol Hepatol 1991;6:35-44.

6. Baxter NN, Virnig DJ, Rothenberger DA, et al. Lymph node evaluation in colorectal cancer patients: a population-based study. J Natl Cancer Inst 2005;97:219-225.

7. Wright FC, Law CH, Last L, et al. Lymph node retrieval and assessment in stage II colorectal cancer: a population based study. Ann Surg Oncol 2003;10:903-909.

8. Colon and Rectum. In: American Joint Committee on Cancer. AJCC Cancer Staging Manual. 6th ed. New York, NY: Springer; 2002:113-124.

9. Taflampas P, Christodoulakis M, Gourtsoyianni S, et al. The effect of preoperative chemoradiotherapy on lymph node harvest after total mesorectal excision for rectal cancer. Dis Colon Rectum 2009;52:14701474 .

10. McDonald JR, Renehan AG, O'Dwyer ST, Haboubi NY. Lymph node harvest in colon and rectal cancer: current considerations. World J Gastorintest Surg 2012;4:9-19.

11. Evans MD, Barton $K$, Rees $A$, et al. The impact of surgeon and pathologist on lymph node retrieval in colorectal cancer and its impact on survival for patients with Dukes' stage B disease. Colorectal Dis 2008;10:157-164.

12. Bilimoria KY, Bentrem DJ, Stewart AK, et al. Lymph node evaluation as a colon cancer quality measure: a national hospital report card. J Natl Cancer Inst 2008;100:1310-1317.

13. Miller ED, Robb BW, Cummings OW, Johnstone PA. The effects of preoperative chemoradiotherapy on lymph node sampling in rectal cancer. Dis Colon Rectum 2012;55:1002-1007.

14. Shen SS, Haupt BX, Ro JY, et al. Number of lymph nodes examined and associated clinicopathologic factors in colorectal carcinoma. Arch Pathol Lab Med 2009;133:781-786.

15. Baxter NN, Morris AM, Rothenberger DA, Tepper JE. Impact of preoperative radiation for rectal cancer on subsequent lymph node evaluation: a population-based analysis. Int J Radiat Oncol Biol Phys 2005;61:426-431

16. Gorog D, Nagy P, Peter A, Perner F. Influence of obesity on lymph node recovery from rectal cancer specimens. Pathol Oncol Res 2003;9:180-183.

17. Nathan $H$, Shore $A D$, Anders RA, et al. Variation in lymph node assessment after colon cancer resection: patient, surgeon, pathologist, or hospital? J Gastrointest Surg 2011;15:471-479.

18. Wong SL, Ji H, Hollenbeck BK, et al. Lymph node examination rates and survival after resection for colon cancer. JAMA 2007;298:2149-2154.

19. Sauer R, Becker $H$, Hohenberger W, et al. Preoperative versus postoperative chemoradiotherapy for rectal cancer. N Engl J Med 2004;351:1731-1740.

20. De la Fuente SG, Manson RJ, Ludwig KA, Mantyh CR. Neoadjuvant chemoradiation for rectal cancer reduces lymph node harvest in proctectomy specimens. J Gastrointest Surg 2009;13:269-274.

21. Marks JH, Valsdottir EB, Rather AA, et al. Fewer than 12 lymph nodes can be expected in a surgical specimen after high-dose chemoradiation therapy for rectal cancer. Dis Colon Rectum 2010;53:1023-1029.

22. Rullier A, Laurent C, Capdepont $M$, et al. Lymph nodes after preoperative chemoradiotherapy for rectal carcinoma: number, status, and impact on survival. Am J Surg Pathol 2008;32:45-50.

23. Bosset JF, Calais G, Mineur L, et al. Fluorouracil-based adjuvant chemotherapy after preoperative chemoradiotherapy in rectal cancer: long-term results of the EORTC 22921 randomised study. Lancet Oncol 2014;15:184-190.

24. Benson AB, Venook AP, Bekaii-Saab T, et al. NCCN Clinical Practice Guidelines in Oncology: Rectal Cancer. Version 2.2016. Accessed March 28, 2016. To view the most recent version of these guidelines, visit NCCN. org. 